\title{
LEER EN CONTEXTOS DE DISTRACCIÓN: EL ROL DE LA INHIBICIÓN Y EL ESTABLECIMIENTO DE OBJETIVOS EN LA COMPRENSIÓN DE TEXTOS ACADÉMICOS DIGITALES
}

\author{
Reading in Distracting Settings: The Role of Inhibition and Setting Goals in \\ the Comprehension of Digital Academic Texts \\ LIRE DANS DES CONTEXTES DISTRACTIFS : LE RÔLE DE L'INHIBITION ET LA FIXATION \\ DES OBJECTIFS DANS LA COMPRÉHENSION DE TEXTES NUMÉRIQUES À L'ACADÉMIE \\ LER EM CONTEXTOS de distração: O PAPEL da INIBIÇÃo E A DEFINIÇão de OBJETIVOS \\ NA COMPREENSÃO DE TEXTOS ACADÊMICOS EM FORMATOS DIGITAIS
}

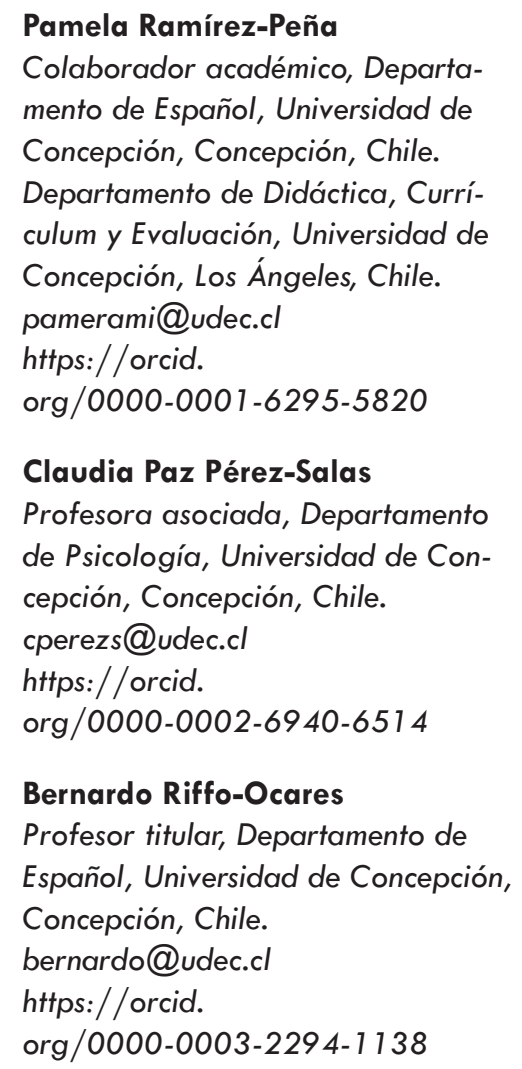

\section{Bernardo Riffo-Ocares}

Profesor titular, Departamento de Español, Universidad de Concepción, Concepción, Chile. bernardo@udec.cl https://orcid. org/0000-0003-2294- 1138

\begin{abstract}
RESUMEN
El texto es la principal herramienta para acceder al conocimiento disciplinar en la educación superior. En la actualidad, las tareas de lectura se desarrollan con frecuencia con textos digitales, lo que implica un entorno de lectura susceptible a la distracción, que requiere de un lector capaz de controlar procesos cognitivos clave para la comprensión. Este artículo reporta los resultados de un estudio piloto experimental, cuyo objetivo fue analizar el efecto de dos funciones ejecutivas: la habilidad de inhibición ante un estímulo distractor y la habilidad de establecimiento de objetivos en la comprensión de textos académicos digitales. Para ello, se empleó la técnica de registro de movimientos oculares durante la lectura. En la prueba participaron 39 estudiantes de primer y segundo año de la carrera de Psicología de una universidad chilena. Los resultados revelaron que, en contextos de distracción, la posibilidad de establecer objetivos a partir de instrucciones de relevancia mejora la comprensión de textos académicos digitales. Se concluye que, en estas situaciones de lectura, disponer de instrucciones de relevancia se asocia con un procesamiento focalizado y eficiente del texto, disminuyendo el efecto negativo, en la comprensión, de elementos ajenos a la tarea.
\end{abstract}

Palabras clave: comprensión lectora; contextos de distracción; educación superior; establecimiento de objetivos; inhibición; textos académicos digitales.

\section{Abstract \\ Written texts are the main tool for accessing disciplinary knowledge in higher education. Nowadays, reading tasks are frequently developed by means of digital texts, which implies a reading environment susceptible to distraction, and requires}

Recibido: 2021-02-17 / Aceptado: 2021-04-28 / Publicado: 2022-02-11

https://doi.org/10.17533/udea.ikala.v27n1a04

Editora: Doris Correa, Universidad de Antioquia, Medellín, Colombia

Derechos patrimoniales, Universidad de Antioquia, 2022. Este es un artículo de acceso abierto, distribuido bajo los términos de la licencia Creative Commons BY-NC-SA 4.0 International. 


\section{Raquel Cerdán-Otero}

Profesora titular, ERI Lectura

(Estructura de Investigación en

Lectura), Facultad de Psicología.

Universidad de Valencia, Valencia,

España.

raquel.cerdan@uv.es

https: //orcid.

org/0000-0002-8283-8995 a reader capable of controlling key cognitive processes in order to adequately understand the content. This article reports the results of an experimental pilot study, which aimed to analyze the effect of two executive functions: The ability to inhibit in the face of a distracting stimulus and the ability to set goals, on the comprehension of digital academic texts. For this purpose, the technique of recording eye movements during reading was used. Thirty-nine Psychology students attending the first and second years at a Chilean university participated in the test. The results revealed that, in contexts of distraction, setting goals based on relevant instructions improves the comprehension of digital academic texts. Findings suggest that in these reading situations clear instructions are associated with a focused and efficient processing of the text, which in turn reduces the negative effect on comprehension from elements outside the task.

Keywords: reading comprehension; distracting contexts; higher education; goal setting; inhibition; digital academic texts.

\section{RÉSUMÉ}

Le texte écrit est le principal outil d'accès aux connaissances disciplinaires dans l'enseignement supérieur. De nos jours, les tâches de lecture sont souvent effectuées avec des textes numériques, ce qui implique un environnement de lecture susceptible d'être distrait, nécessitant un lecteur capable de contrôler les processus cognitifs clés pour la compréhension. Cet article rapporte les résultats d'une étude pilote expérimentale dont l'objectif était d'analyser l'effet de deux fonctions exécutives : la capacité d'inhibition face à un stimulus distrayant et la capacité de se fixer des objectifs, sur la compréhension de textes académiques numériques. Pour ce faire, la technique d'enregistrement des mouvements oculaires pendant la lecture a été utilisée. Trente-neuf étudiants en première et deuxième année de psychologie d'une université chilienne ont participé au test. Les résultats ont révélé que, dans des contextes de distraction, la possibilité de fixer des objectifs basés sur des instructions pertinentes améliore la compréhension de textes académiques numériques. Il est conclu que dans ces situations de lecture, le fait de disposer d'instructions pertinentes est associé à un traitement ciblé et efficace du texte, réduisant l'effet négatif sur la compréhension des éléments étrangers à la tâche. Les implications de ces résultats sont discutées et les futures questions de recherche sont projetées.

Mots clés : compréhension de la lecture ; contextes de distraction ; enseignement supérieur ; fixation d'objectifs ; inhibition ; textes académiques numériques.

\section{RESUMO}

O texto escrito é o principal instrumento de acesso ao conhecimento disciplinar no ensino superior. Hoje, as tarefas de leitura são mais frequentemente realizadas sobre textos digitais, o que implica um ambiente de leitura suscetível de distração, o que exige do leitor controlar processos cognitivos chave para garantir a compreensão do conteúdo. Este artigo relata os resultados de um estudo piloto experimental, cujo objetivo era analisar o efeito de duas funções executivas: a capacidade de inibição na frente de um estímulo perturbador e a capacidade de definir objetivos, para a compreensão de textos académicos digitais. Para este fim, nós utilizamos a técnica de registro de movimentos oculares durante a leitura. Trinta e nove estudantes de Psicologia do primeiro e segundo anos de uma universidade chilena participaram no teste. Os resultados revelaram que, em contextos de distração, a possibilidade de definir objetivos com base em instruções 
relevantes melhora a compreensão de textos académicos digitais. Conclui-se que, nestas situações de leitura, ter instruções relevantes está associado a um processamento focalizado e eficiente do texto, o qual reduz o efeito negativo, na compreensão, de elementos estranhos da tarefa.

Palavras-chave: compreensão de leitura; contextos de distração; ensino superior; definiçã de objetivos; inibição; textos académicos digitais. 


\section{Introducción}

Desde una perspectiva comportamental, la comprensión del texto escrito se conceptualiza como una actividad intencional y voluntaria, en la cual los lectores actúan según las características del texto, los objetivos de lectura y el tipo de tarea a desarrollar a partir de la lectura (Snow, 2002). Esta aproximación al fenómeno de la comprensión lectora da cuenta de nuevas formas de abordar los procesos involucrados en la lectura, debido a la diversidad de contextos y tareas de lectura existentes en la actualidad (Parodi et al., 2020).

La comprensión lectora está determinada por habilidades específicas y generales. Las primeras corresponden a las habilidades lingüisticas, que se refieren a los procesos necesarios para descifrar el código escrito (p. ej. procesamiento fonológico, ortográfico, reconocimiento de palabras) y tienden a automatizarse con la práctica y la experiencia lectora, lo que permite liberar recursos cognitivos para un procesamiento profundo del texto. Por otro lado, las habilidades cognitivas corresponden a habilidades generales que incluyen los procesos requeridos para construir una representación mental coherente a partir de la información textual (p. ej. vocabulario, inferencias, memoria de trabajo, entre otras) y que posibilitan comprender conceptos e ideas implicadas en el texto (CanetJuric et al., 2009; Kintsch, 1998). Al respecto, existe evidencia que sostiene que las habilidades cognitivas podrían llegar a ser predominantes para distinguir entre buenos y malos lectores en estudiantes de mayor edad, dado que en este grupo las habilidades lingüísticas han sido automatizadas gracias a la experiencia lectora (Potocki et al., 2017; Vellutino et al., 2007).

El contexto de lectura de textos académicos no ha estado ajeno a la incorporación de dispositivos digitales que incluyen, cada vez con mayor frecuencia, el uso de textos digitales (Ramírez-Peña, 2020). Estos, junto con el acceso masivo a internet y a las nuevas tecnologías, han generado nuevos contextos de lectura, en los cuales compiten estímulos diversos (Delgado et al., 2018; Salmerón y Delgado, 2019) que tienden a generar desorientación y un procesamiento superficial del texto (Piovano et al., 2018).

En esta situación, las habilidades cognitivas desempeñan un rol fundamental, pues el despliegue de procesos como la inhibición permite suprimir estímulos que compiten durante el desarrollo de una tarea determinada (Diamond, 2013), y establecer objetivos en relación con la tarea posibilita enfocar la atención de manera eficiente y estratégica (Anderson, 2002; Ramírez-Peña, 2020). Dichas habilidades cognitivas forman parte del constructo denominado "funciones ejecutivas" (FE), cuya relación con la lectura ha sido ampliamente abordada en los últimos 20 años (Follmer, 2017; Ramírez-Peña, 2020; Roldán, 2016), aunque en su mayoría en población en edad escolar (Georgiou y Das, 2016).

Las FE comprenden un conjunto de procesos cognitivos que actúan de manera interrelacionada $\mathrm{e}$ intervienen en el control voluntario de conductas, emociones y pensamientos en función de objetivos específicos (Friedman y Miyake, 2017). Estos procesos son disociables (Anderson, 2002), operan en situaciones que implican un elevado esfuerzo cognitivo y, en su conjunto, se definen como procesos de control cognitivo (Diamond, 2013).

Pese a que existe abundante evidencia empírica sobre la relación entre las FE y la comprensión lectora, la investigación ha sido escasa en Latinoamérica (Roldán, 2016) y los resultados parecen no ser concluyentes (Follmer, 2017). Por ello, el presente estudio tuvo como objetivo analizar el efecto de dos FE (inhibición y establecimiento de objetivos) en la comprensión de textos académicos digitales en lectores que se encuentran cursando primer y segundo año de educación superior en una universidad chilena.

Las hipótesis de trabajo que guiaron el estudio fueron las siguientes: una hipótesis de efectos principales que plantea que los lectores obtienen un 
mejor puntaje en una prueba de comprensión cuando 1) son capaces de inhibir la mirada a un distractor o resistir la interferencia distractora; y 2) son expuestos a instrucciones de relevancia antes de leer el texto; y, una hipótesis de interacción, que sugiere que la inhibición y el establecimiento de objetivos tienen un efecto conjunto sobre la comprensión, lo que se evidencia como un efecto de interacción entre las variables antes mencionadas sobre la comprensión de un texto académico digital (Ramírez-Peña, 2020).

A continuación se presenta la evidencia empírica existente sobre la relación entre las FE de inhibición y establecimiento de objetivos, y la comprensión lectora. Posteriormente, se expone la metodología empleada para el desarrollo del presente estudio y los resultados obtenidos. Por último, se discuten los resultados a la luz de la evidencia y se concluye cuál es el rol de las FE de inhibición y establecimiento de objetivos en la comprensión de textos académicos digitales.

\section{Marco teórico}

En esta sección se presenta la evidencia que existe sobre la relación entre las variables de estudio. Para ello, se definen las variables "inhibición" y "establecimiento de objetivos", y se muestra la relación de cada una de ellas con la comprensión lectora.

\section{Inhibición y comprensión lectora}

En adultos sanos, las diferencias individuales relacionadas con la inhibición han sido consideradas una de las causas que subyacen a las dificultades en comprensión lectora (De Beni et al., 1998; Gernsbacher, 1993). La inbibición es un proceso cognitivo que permite suprimir estímulos externos e internos que pueden dificultar o interferir en el funcionamiento cognitivo; o bien, suprimir respuestas automáticas que son irrelevantes para el logro de una tarea (Diamond, 2013). En el ámbito de la lectura, la inhibición actúa como un mecanismo general que se ocupa del control de las interferencias tanto lingüísticas como contextuales durante el procesamiento de un texto (Canet-Juric et al., 2009; Ramírez-Peña, 2020).

El control inhibitorio desempeña tres funciones: eliminación de la respuesta automática (capacidad de anular una respuesta automática), rechazo de la interferencia distractora (capacidad de suprimir información irrelevante) y resistencia a la interferencia proactiva (capacidad de cancelar el recuerdo de información relevante para tareas previas) (Friedman y Miyake, 2004). En este estudio, la inhibición se midió de manera indirecta, mediante la presencia de distractores que aparecen como ventanas emergentes $(p o p-u p)$ durante la lectura, dado que investigaciones previas sostienen que mantener la atención en información relevante para una tarea mientras se ignora un elemento distractor representa una alta exigencia cognitiva, especialmente durante periodos extensos de tiempo (Annerer-Walcher et al., 2020). Dependiendo de las características de la tarea y los recursos cognitivos, la atención puede variar desde una absorción profunda hasta una susceptibilidad considerable a la distracción (Wilcockson et al., 2019), lo que podría estar asociado con el rendimiento en tareas que implican la comprensión de un texto.

El uso del registro de movimientos oculares para explorar la inhibición ante un distractor ha sido utilizado en estudios previos, con el objetivo de resguardar la validez ecológica de la evaluación en situaciones de lectura en las que se requiere que el lector mantenga focalizada la atención en el procesamiento del texto mientras intenta inhibir la mirada o atención hacia el estímulo que compite (Ramírez-Peña, 2020; Wilcockson et al., 2019). Según los hallazgos, ignorar distractores parece dar cuenta de la habilidad de control inhibitorio (Gaspelin y Luck, 2018; Rozek et al., 2012; Wilcockson et al., 2019). No obstante, los resultados de estudios previos no son concluyentes en cuanto a la relación entre la habilidad de inhibición y la comprensión lectora, debido, probablemente, a la diversidad de instrumentos con los que se evalúan ambos procesos cognitivos, la función del control inhibitorio evaluada o la etapa 
de desarrollo cognitivo de la población evaluada (Arrington et al., 2014; Borella y De Ribaupierre, 2014; Borella et al., 2010; Canet-Juric et al., 2009, 2013; Demagistri et al., 2014; Follmer, 2017; Ramírez-Peña, 2020; Roldán, 2016).

\section{Establecimiento de objetivos y comprensión lectora}

Este dominio de las FE corresponde a un proceso cognitivo que permite planificar acciones de manera anticipada y enfocar la atención de forma eficiente y estratégica. Las dificultades en este dominio afectan la capacidad de resolución de problemas, reflejada en desorganización, dificultades en el desarrollo de estrategias eficientes y un pobre razonamiento conceptual (Anderson, 2002).

Definir objetivos de lectura es parte importante del proceso de lectura en un contexto de lecturaorientada-a-tareas (Vidal-Abarca et al., 2011). Un lector competente define objetivos de lectura desde la interpretación que hace de las tareas y a partir de claves de relevancia que se desprenden de las instrucciones. Como consecuencia de ello, solo aquella información del texto que es relevante para cumplir con el objetivo lo será también para el lector (McCrudden, 2011; McCrudden y Schraw, 2007). Este tipo de lector, por lo tanto, focalizará su atención en el procesamiento de aquellos segmentos textuales que contienen información relevante para la tarea a desarrollar (Cerdán et al., 2013; León et al., 2019). En este estudio se emplearon instrucciones de relevancia, con el objetivo de gatillar un procesamiento focalizado y eficiente del texto, permitiendo que el lector definiera objetivos a partir de las instrucciones (McCrudden y Schraw, 2007).

La evidencia muestra que los lectores que son expuestos a instrucciones de relevancia centran su atención de manera selectiva en las partes del texto significativas para la tarea (Cerdán et al., 2013; León et al., 2019). De manera específica, en un estudio con textos de ciencias en el que participaron estudiantes de sexto grado (Media —M-: 12,36 años), se observó que los estudiantes que reciben instrucciones de lectura realizan un esfuerzo cognitivo mayor y reportan mayores tiempos de lectura que los estudiantes que no reciben instrucciones (Jian, 2018). Esto podría relacionarse con las características de los lectores estratégicos, quienes seleccionan y mantienen en mente sus objetivos de lectura, generando medios adecuados para lograrlos (Georgiou y Das, 2016).

Un modelo de procesamiento del texto centrado en objetivos clasifica las instrucciones de relevancia en 1) instrucciones de relevancia general y 2) instrucciones de relevancia específica (McCrudden y Schraw, 2007). Las primeras explicitan temas, propósitos o contextos generales de lectura (contestar preguntas después de leer, escribir un resumen a partir del texto, entre otras); las segundas, en cambio, se enfocan en elementos específicos del texto que son relevantes para la tarea (Cerdán et al., 2019; McCrudden y Schraw, 2007; Rapp y McCrudden, 2018).

\section{Método}

A continuación se explica el diseño de la investigación que se reporta en este estudio, los participantes, instrumentos, materiales y la forma en que se desarrolló la tarea experimental.

\section{Diseño}

Esta investigación se desarrolló como un estudio piloto de tipo experimental, con diseño factorial mixto de $2 \times 2$ (Balluerka y Vergara, 2002). El factor intrasujetos fue la variable "inhibición” y el factor intersujetos, la variable "establecimiento de objetivos".

El primer factor originó dos condiciones experimentales (lectura con distractor y lectura sin distractor), y el segundo factor, dos grupos experimentales (grupo con instrucciones previas a la lectura - GCI- y grupo sin instrucciones previas a la lectura - GSI-). La variable dependiente 
fue “comprensión lectora”, operacionalizada como el puntaje de cada participante en la prueba de lectura.

Se realizó un contrabalanceo de los textos, una estrategia de control experimental con el fin de descartar un efecto de posición en los resultados. Como se trató de dos textos, algunos participantes partieron la tarea leyendo el texto 1 , y otros, el texto 2. A su vez, se contrabalanceó la presentación de los distractores: a un grupo se les presentó en el primer texto, y a otros, en el segundo. Luego, se efectuó un análisis que permitió comprobar la ausencia de un efecto de posición de las condiciones con distractor/sin distractor y la ausencia de efecto de posición de los textos.

\section{Participantes}

Participaron, en este estudio, 39 estudiantes de Psicología de una universidad tradicional de la ciudad de Concepción (Chile) (53,8 \% mujeres; $M=19,5$ años, desviación estándar $-D E-=1,27)$. La muestra seleccionada fue

72 intencional, no probabilística, de acuerdo con los siguientes criterios de inclusión: cursar primer o segundo año del programa de estudios, sin diagnóstico previo de dificultades lectoras y presentar visión normal o corregida.
Los participantes fueron asignados de manera aleatoria a cada grupo: 1) grupo sin instrucciones de relevancia (GSI, $n=19$ ) y 2 ) grupo con instrucciones de relevancia (GCI, $n=20)$.

\section{Instrucciones}

Para evaluar el efecto del establecimiento de objetivos, en este caso "objetivos de lectura”, se manipuló la variable "instrucciones previas a la lectura", con dos condiciones: con instrucciones de relevancia y sin instrucciones de relevancia. Así, a uno de los grupos se le otorgó instrucciones de relevancia para la lectura, que aludían directamente a la necesidad de contestar un conjunto de preguntas para cada uno de los textos, mientras que al otro grupo se le presentó una consigna general ("Lee atentamente el siguiente texto"), que no entregaba pistas concretas acerca de la tarea de lectura o de las preguntas. En la Tabla 1 se muestran las dos consignas asignadas.

\section{Textos}

Para la tarea de lectura, se utilizaron dos textos académicos, extraídos de una prueba estandarizada para evaluar la comprensión lectora, denominada Lectum 7 (Véliz et al., 2013a, 2013b).

Tabla 1 Consigna grupos experimentales

\begin{tabular}{l}
\hline \multicolumn{1}{c}{ Con instrucciones de relevancia } \\
\hline Lee el texto que se presenta a continuación para contestar \\
preguntas de comprensión al final de la lectura. \\
- ¿Cuál es la finalidad del crecimiento poblacional? \\
- ¿Qué es la resistencia ambiental? \\
- ¿Por qué se hace referenciones de relevancia a las bacterias en el texto? \\
¿Qué características tiene el modelo logístico de crecimiento \\
de una población? \\
¿Cuál es el propósito del autor del texto? \\
Para la lectura de gráficos, considera las siguientes \\
preguntas: \\
¿Qué función cumplen los gráficos en el texto? \\
¿Qué información entrega cada uno de ellos?
\end{tabular}


Esta prueba consta de dos formas equivalentes, lo que permitió contar con dos textos (y sus respectivas preguntas de comprensión), que componen un instrumento validado para evaluar comprensión lectora en población que está finalizando su enseñanza secundaria (véase Riffo et al., 2013, para detalles sobre el proceso de validación de los textos).

En ambos textos predomina la secuencia explicativa y se estructuran en torno a un tema especializado del área de las ciencias naturales y de las ciencias sociales, empleando segmentos textuales continuos y discontinuos (gráficos).

\section{Distractores}

Se usaron dos distractores para evaluar el efecto de la inhibición en la comprensión de un texto académico digital. Los distractores se seleccionaron a partir del resultado de un breve estudio normativo efectuado por una de las investigadoras el año anterior al estudio, en el cual, mediante una encuesta, se solicitó a un grupo de 50 estudiantes de primer año, distintos de los del presente estudio, ordenar, mediante un número, los estímulos presentados, señalando con el número 1 aquel que, al aparecer durante la lectura, con mayor probabilidad podría generar distracción, hasta llegar al número 7 , que representa una menor probabilidad de distracción. Tras ese estudio, se seleccionaron los dos estímulos que fueron ubicados en el primer y el segundo lugar de probabilidad de generar distracción durante la lectura.

Para aumentar la probabilidad de interferencia durante la lectura, se emplearon dos distractores distintos en contenido, pero equivalentes en estructura. El distractor 1 informaba sobre el caso de una niña de 3 años, cuyo coeficiente intelectual superó al de Albert Einstein; el segundo distractor, sobre una nueva tecnología para optimizar la señal de wifi en casa (véase Figura 1). Ambos distractores se presentaron en posiciones distintas (izquierda, derecha) y con segmentos textuales diferentes (gráfico, párrafo).

\section{Instrumentos}

Para recolectar los datos, se empleó un eye tracker y una prueba de comprensión lectora. El eye tracker permitió registrar los movimientos oculares de los participantes al aparecer los distractores durante la lectura. Con ello, el tiempo de mirada al distractor se consideró un proxy de la variable "inhibición". Para conocer el rendimiento alcanzado en la lectura de cada uno de los textos presentados en la

Figura 1 Estructura de los distractores empleados en la tarea experimental.
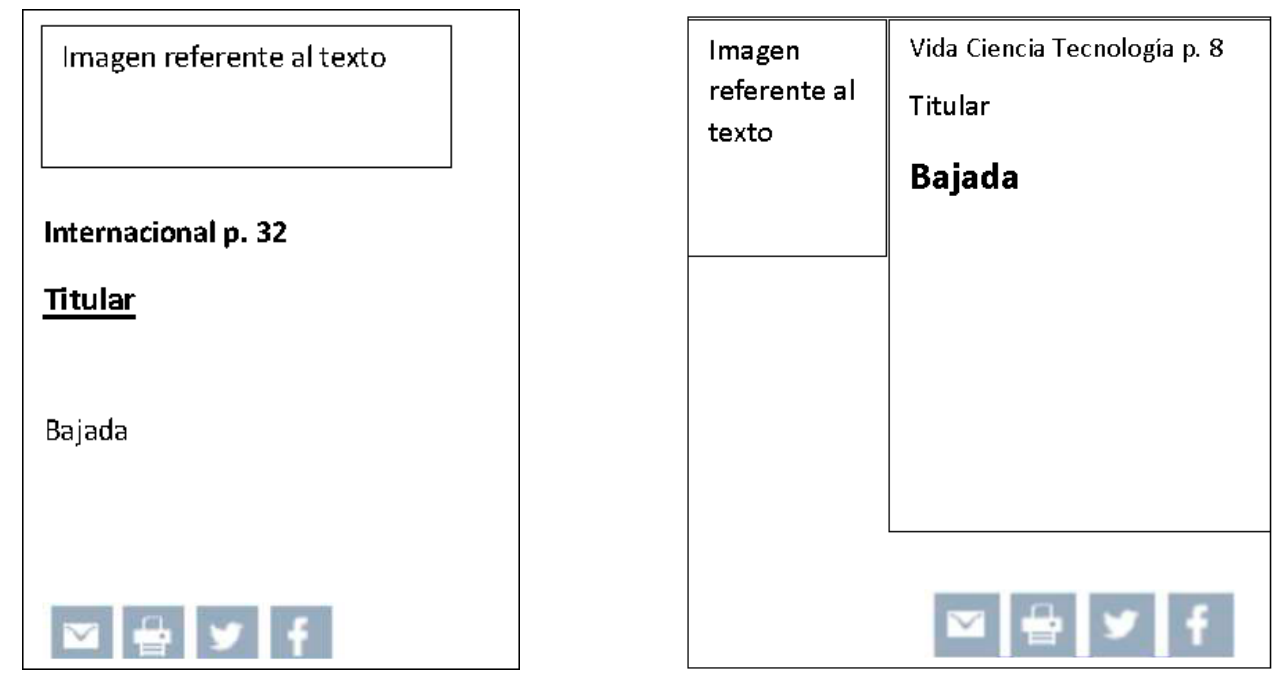

Nota: Ambos distractores son equivalentes en cuanto a su estructura: presentan una imagen, titular y bajada. "Bajada": subtítulo o entrada del texto original. 
tarea experimental, se empleó una prueba de comprensión lectora por cada texto.

- Equipo de registro ocular. Se empleó el equipo SMI RED 500. El texto se presentó en un monitor LCD de 22 pulgadas, con una resolución de $1680 \times 1050$. La frecuencia de muestreo utilizada fue de $500 \mathrm{~Hz}$. Se usó el programa Experiment Center para el diseño de la tarea experimental y los datos se extrajeron con el programa BeGaze, ambos desarrollados por la empresa SensoMotoric Instruments para operar con el SMI RED 500.

- Prueba de comprensión lectora. Se presentaron 10 preguntas de selección múltiple para evaluar la comprensión de cada texto, extraídas de la prueba estandarizada Lectum 7, formas A $(\alpha=0,87)$ y B $(\alpha=0,83)$. El índice de confiabilidad de las formas paralelas es de 0,77 (Riffo et al., 2013).

\section{Tareas}

Para conocer el efecto de la inhibición y del establecimiento de objetivos, se formularon cuatro tareas experimentales, generadas por la manipulación de ambas variables (véase Figura 2). Cada sujeto fue asignado a un grupo (con o sin instrucciones de relevancia) y llevó a cabo dos tareas experimentales (lectura con o sin distractores). Para evaluar la comprensión, se aplicó una prueba sobre cada texto leído inmediatamente después

Figura 2 Tareas experimentales.
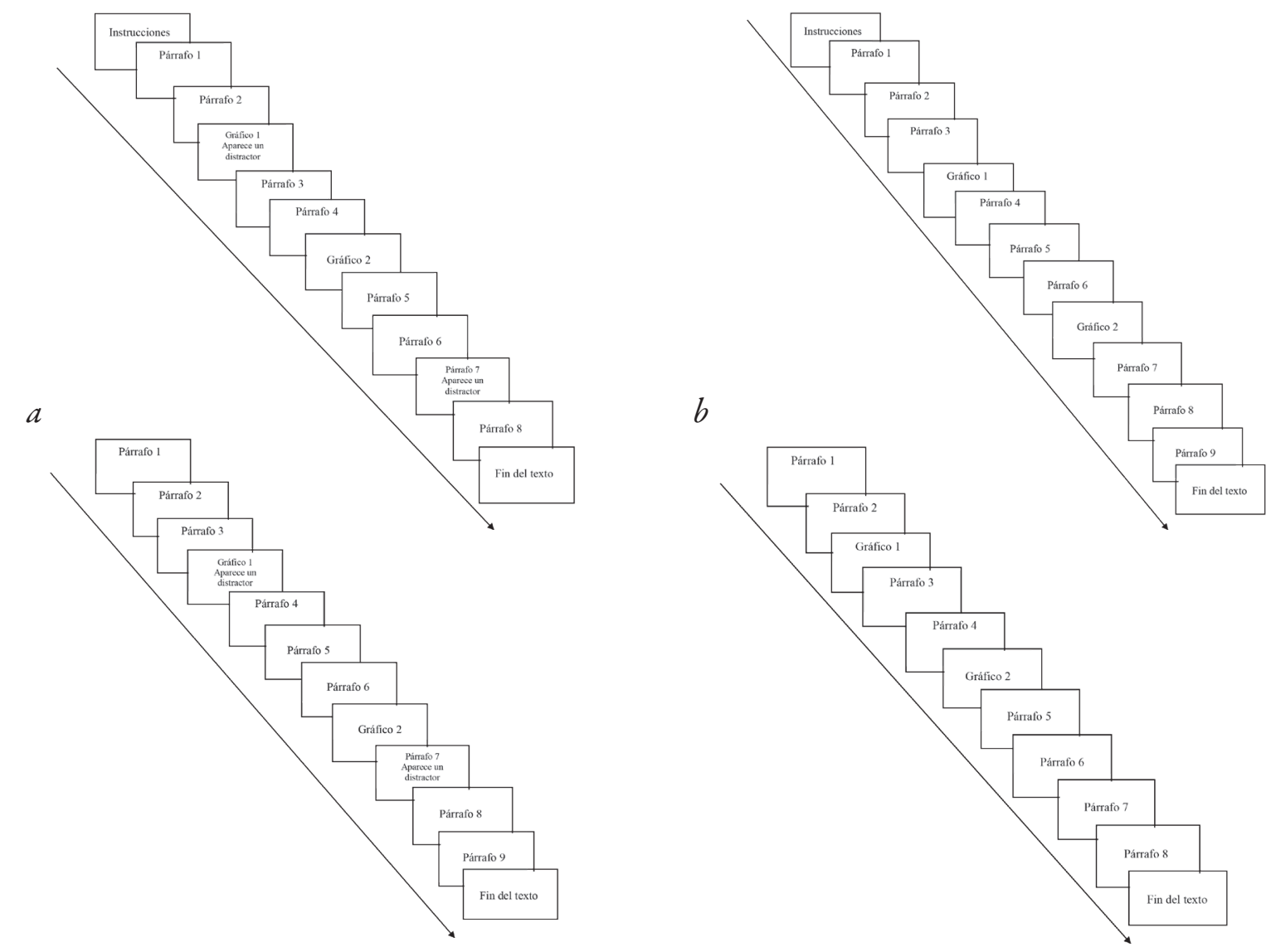

c

$d$

Nota: $a$ y $b$ corresponden al grupo con instrucciones de relevancia (GCI); $c$ y $d$, al grupo sin instrucciones de relevancia (GSI). Condiciones $a$ y $c$ tienen tareas con distractores, mientras las condiciones $b$ y $d$ no presentan distractores. 
de finalizar la lectura. A continuación se describe cada una de las tareas.

- GCI lectura de texto con distractor. La tarea comenzó con la lectura de instrucciones de relevancia, las que se presentaron en la pantalla con tiempo indefinido. Cada participante podía decidir leer o no las instrucciones nuevamente. Para ello, debía contestar seleccionando la opción "sí" o "no": si la respuesta era afirmativa, se presentaban nuevamente las preguntas en la pantalla con tiempo indefinido; en caso de responder "no", se daba inicio a la lectura del texto y aparecía de inmediato el primer párrafo del texto.

La tarea de lectura consistió en leer un texto digital que se presenta segmentado en párrafos. Se mostró un párrafo por pantalla; en el caso de los gráficos, se ubicaron al centro de la pantalla, sin texto e inmediatamente después del párrafo que hacía referencia a ellos. Para avanzar en la lectura, el participante debía presionar la barra espaciadora del teclado.

La lectura del texto se interrumpió en dos oportunidades por un distractor: la primera vez, durante la lectura de un párrafo, y la segunda, durante la lectura de un gráfico. El primer distractor apareció en la parte inferior izquierda de la pantalla, y el segundo, en la parte inferior derecha. En ambos casos, los distractores aparecieron como ventanas emergentes sin movimiento y permanecieron visibles por 2000 ms (véanse Figuras 3 y 4).

Al terminar la lectura en cada tarea experimental, se presentó la opción de volver a leer el texto completo.

- GCI lectura de texto sin distractor. Al igual que en la condición anterior, el participante leyó instrucciones de relevancia sobre un segundo texto antes de comenzar la lectura del mismo. La diferencia con la tarea anteriormente descrita radica en que durante la lectura de este texto no aparecen distractores.
Figura 3 Aparición de un distractor durante la lectura de un gráfico.

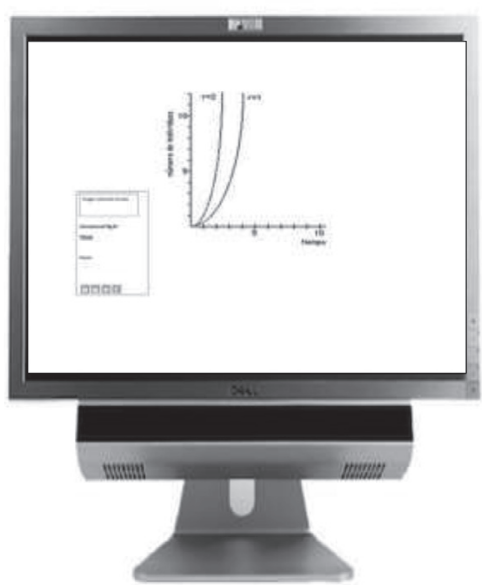

Figura 4 Aparición de un distractor durante la lectura de un párrafo.

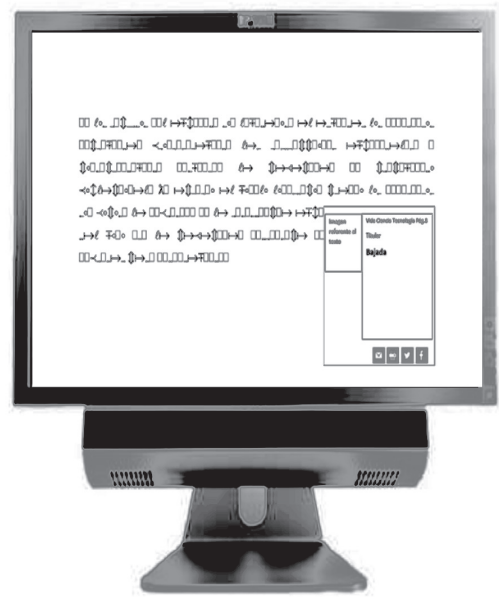

- GSI lectura de texto con distractor. Para esta tarea se empleó el texto que en el GCI se presenta sin distractores, con el objetivo de contar con un diseño equilibrado a través del contrabalanceo de los textos. La tarea se inició de inmediato con la lectura de un texto digital que se presenta segmentado en párrafos (solo aparece una consigna general antes de iniciar la lectura). Al igual que en el GCI, se mostró un párrafo por pantalla; en el caso de los gráficos, se ubicaron en el centro de la pantalla, sin texto e inmediatamente después del párrafo que hace referencia a ellos. La aparición de los 
distractores ocurrió de la misma forma que en el GCI.

- GSI lectura de texto sin distractor. Para esta tarea, se empleó el texto que en el GCI se presenta con distractores. $\mathrm{Al}$ igual que en la condición anterior, la tarea comenzó de inmediato con la lectura de un texto digital que se segmenta en párrafos.

\section{Procedimiento}

La investigación fue aprobada por el Comité de Ética, Bioética y Bioseguridad de la Vicerrectoría de Investigación de la Universidad de Concepción (Chile). Cada participante firmó un consentimiento informado, y como incentivo a la participación, recibió la bonificación de un punto para una evaluación en la asignatura de Neuropsicología.

La ejecución de la tarea experimental se desarrolló de manera individual en un ambiente de laboratorio. Al comenzar la sesión, se solicitó a cada participante la lectura y firma del consentimiento informado. A continuación, la investigadora explicó el procedimiento para el desarrollo del experimento y solicitó al participante ubicarse frente al monitor donde se presentó la tarea experimental. La distancia entre el participante y el monitor osciló entre los 60 y $67 \mathrm{~cm}$, dependiendo de la calibración obtenida (valores en las coordenadas $x, y<1)$.

Los participantes fueron asignados a cada grupo al azar, resguardando el cumplimiento del contrabalanceo de la condición con/sin distractores.

Cada condición experimental comenzó con una calibración. Los participantes leyeron dos textos: uno con distractores y otro sin distractores. El tiempo de lectura fue indefinido y autoadministrado por cada participante. Una vez finalizada la lectura de un texto, cada participante rindió una prueba de comprensión sobre el texto, aplicada en formato papel y lápiz, con tiempo indefinido. $\mathrm{Al}$ finalizar la prueba, se solicitó al participante ubicarse nuevamente frente al monitor para la lectura del segundo texto. Se procedió a la calibración respectiva y se inició la lectura del segundo texto. Finalmente, el participante rindió una prueba de comprensión sobre el segundo texto. El tiempo total por participante osciló entre 45 y 60 minutos.

\section{Análisis de los datos}

Se efectuaron dos análisis de varianza (ANOVA factorial de medidas repetidas), previa exploración de los datos (véanse Tabla 2) y comprobación de los supuestos de normalidad y homocedasticidad. El primer análisis tuvo como objetivo comprobar el efecto de distracción de los dos distractores que aparecen durante la lectura de uno de los textos, empleando la medida "tiempo de mirada" como variable dependiente, "inhibición" como factor intrasujetos y "establecimiento de objetivos" como factor intersujetos. Se realizó un segundo análisis para conocer el efecto de la inhibición y el establecimiento de objetivos en la comprensión de textos académicos digitales. El factor intrasujetos fue "inhibición” y el factor intersujetos fue "establecimiento de objetivos". La medida dependiente fue el puntaje obtenido en las pruebas de comprensión de cada texto (con/sin distractor).

Tabla 2 Estadísticos descriptivos: comprensión de textos académicos digitales

\begin{tabular}{|c|c|c|c|c|}
\hline \multirow{2}{*}{ Grupo } & \multirow{2}{*}{ Condición } & \multirow{2}{*}{$\begin{array}{l}\text { Media } \\
\text { (DE) }\end{array}$} & \multicolumn{2}{|c|}{$\begin{array}{c}\text { Intervalo de } \\
\text { confianza al } 95 \%\end{array}$} \\
\hline & & & $\begin{array}{l}\text { Límite } \\
\text { inferior }\end{array}$ & $\begin{array}{l}\text { Límite } \\
\text { superior }\end{array}$ \\
\hline $\begin{array}{l}\text { Sin } \\
\text { instrucciones }\end{array}$ & $\begin{array}{l}\text { Con } \\
\text { distractor }\end{array}$ & $\begin{array}{c}5,32 \\
(0,40)\end{array}$ & 4,50 & 6,13 \\
\hline $\begin{array}{l}\text { de relevancia } \\
(\mathrm{n}=19)\end{array}$ & $\begin{array}{l}\text { Sin } \\
\text { distractor }\end{array}$ & $\begin{array}{c}6,79 \\
(0,38)\end{array}$ & 6,02 & 7,56 \\
\hline $\begin{array}{l}\text { Con } \\
\text { instrucciones }\end{array}$ & $\begin{array}{l}\text { Con } \\
\text { distractor }\end{array}$ & $\begin{array}{l}7,35 \\
(0,39)\end{array}$ & 6,55 & 8,15 \\
\hline $\begin{array}{l}\text { de relevancia } \\
(\mathrm{n}=20)\end{array}$ & $\begin{array}{l}\text { Sin } \\
\text { distractor }\end{array}$ & $\begin{array}{c}5,80 \\
(0,37)\end{array}$ & 5,05 & 6,55 \\
\hline
\end{tabular}

DE: desviación estándar. 
Tabla 3 Estadísticos descriptivos: duración de la mirada en distractores empleados en el estudio

\begin{tabular}{lccccc}
\hline & $\begin{array}{c}\text { Media } \\
\text { (DE) }\end{array}$ & Mínimo & Máximo & $\mathbf{t}$ & $\begin{array}{c}\text { Sig. } \\
\text { (bilateral) }\end{array}$ \\
\hline Duración de la mirada & $\begin{array}{c}763,85 \mathrm{~ms} \\
(613,74)\end{array}$ & 0 & $1838,8 \mathrm{~ms}$ & 777 & $<0,001$ \\
en distractor 1 & $\begin{array}{c}407,326 \mathrm{~ms} \\
\text { Duración de la mirada } \\
\text { en distractor 2 }\end{array}$ & 0 & $1310,4 \mathrm{~ms}$ & 659 & $<0,001$ \\
\hline
\end{tabular}

DE: desviación estándar; sig.: nivel de significación estadística; valor t: comparación de medias.

\section{Resultados}

El análisis de datos consideró dos momentos que se evidencian en dos análisis. Dado que se trató de un estudio piloto, el primer análisis se realizó con el objetivo de comprobar el efecto de los distractores durante la lectura. El segundo corresponde al análisis principal del estudio y el objetivo fue comprobar las hipótesis de trabajo mencionadas previamente. Los resultados de cada análisis se muestran a continuación.

\section{Primer análisis: tiempo de mirada en los distractores}

Previo al análisis principal de este estudio, se comprobó si los lectores miraron los distractores y, en caso de hacerlo, por cuánto tiempo (véase Tabla 3 ). La medida estadística utilizada fue la duración de la mirada sobre el distractor, entendida como la suma de todas las fijaciones y sacadas sacadas oculares (movimientos oculares rápidos entre dos punto de fijación) desde el ingreso al distractor, es decir, desde que el lector reacciona ante el estímulo que interrumpe la lectura (sacada ocular de ingreso) hasta el momento en que vuelve al texto (sacada ocular de salida), considerando el tiempo que permanece mirando el distractor (sacadas y fijaciones en el distractor). Cada distractor corresponde a un área de interés.

Los resultados indican que el $76,9 \%$ de los sujetos miró el distractor 1 y el 71,9 \% miró el distractor 2. Al explorar los datos, se observó que quienes no miraban el primer distractor luego miraban el segundo distractor. Por lo tanto, se evidencia el efecto del distractor sobre la atención del lector. El análisis de varianza permitió detectar diferencias estadísticamente significativas en el tiempo de mirada de ambos distractores $(F(1,37)=7,87$, $p=0,01, \eta^{2}=0,18$, donde $F$ es un estadístico que da cuenta de la variación entre las medias; $p$ es el nivel de significación - en este caso, la variación es significativa, ya que el valor es menor que 0,05-, y $\eta^{2}$ es el coeficiente eta al cuadrado - a partir de este coeficiente, se infiere el tamaño del efecto-). No obstante, no se detectaron diferencias estadísticamente significativas en el tiempo de mirada a ambos distractores entre los grupos $(F(1,37)=0,04, p=0,85)$.

\section{Segundo análisis: efecto de la inhibición y el establecimiento de objetivos en la comprensión de textos académicos digitales}

Se analizaron el efecto de los factores grupo (GCI, GSI), la condición de lectura (texto con distractores, texto sin distractores) y la interacción entre ambos. La prueba ANOVA factorial de medidas repetidas indica que existe una interacción significativa entre los factores presencia de distractor y grupo sobre la comprensión de un texto académico $\operatorname{digital}(F(1,37)=19,198, p<0,001, \eta 2=0,342)$.

Los resultados dan cuenta de un efecto de magnitud media, siendo el efecto de interacción significativo (véase Tabla 4). 
Tabla 4 Efecto de la inhibición y el establecimiento de objetivos sobre la comprensión de textos académicos digitales

\begin{tabular}{|c|c|c|c|c|c|c|c|c|}
\hline & $\begin{array}{l}\text { Tipo III de } \\
\text { suma de } \\
\text { cuadrados }\end{array}$ & gl & $\begin{array}{c}\text { Media } \\
\text { cuadrática }\end{array}$ & $\mathbf{F}$ & Sig. & $\eta^{2}$ & $\begin{array}{l}\text { Parámetro } \\
\text { sin } \\
\text { centralidad }\end{array}$ & $\begin{array}{l}\text { Potencia } \\
\text { observada }\end{array}$ \\
\hline $\begin{array}{l}\text { Factor intersujetos } \\
\text { (Establecimiento de } \\
\text { objetivos) }\end{array}$ & 5,32 & 1 & 5,32 & 1,50 & 0,23 & 0,04 & 1,50 & 0,22 \\
\hline $\begin{array}{l}\text { Factor intrasujetos } \\
\text { (Inhibición) }\end{array}$ & 0,03 & 1 & 0,03 & 0,01 & 0,91 & $<0,001$ & 0,01 & 0,05 \\
\hline $\begin{array}{l}\text { Interacción } \\
\text { Inhibición × Objetivos }\end{array}$ & 44,54 & 1 & 44,54 & 19,15 & $<0,001^{*}$ & 0,34 & 19,20 & 0,99 \\
\hline $\begin{array}{l}\text { Error } \\
\text { (Factor intersujetos) }\end{array}$ & 131,17 & 37 & 3,55 & & & & & \\
\hline $\begin{array}{l}\text { Error } \\
\text { (Factor intrasujetos) }\end{array}$ & 85,84 & 37 & 2,32 & & & & & \\
\hline
\end{tabular}

El efecto de las instrucciones según la condición de lectura (con/sin distractor) se analizó a partir del puntaje que alcanzó la muestra en las pruebas de lectura para cada condición. Las diferencias de puntaje en comprensión lectora revelan que, en una situación de lectura con distractores, el grupo expuesto a instrucciones antes de leer obtiene un mejor desempeño en comprensión de textos académicos que el grupo que no fue expuesto a instrucciones antes de leer. Específicamente, en la condición sin distractor, disponer o no de instrucciones de relevancia antes de leer no genera diferencias estadísticamente significativas en el puntaje en comprensión lectora $(F(1,37)=3,45, p>0,05)$. Sin embargo, en la condición con distractor, disponer de instrucciones de relevancia general y específica facilita el desempeño en la tarea $(F(1,37)=13,011, p=0,01)$ (véase Figura 5).

Figura 5 Efecto de interacción de las variables inhibición y establecimiento de objetivos sobre la comprensión de textos académicos digitales

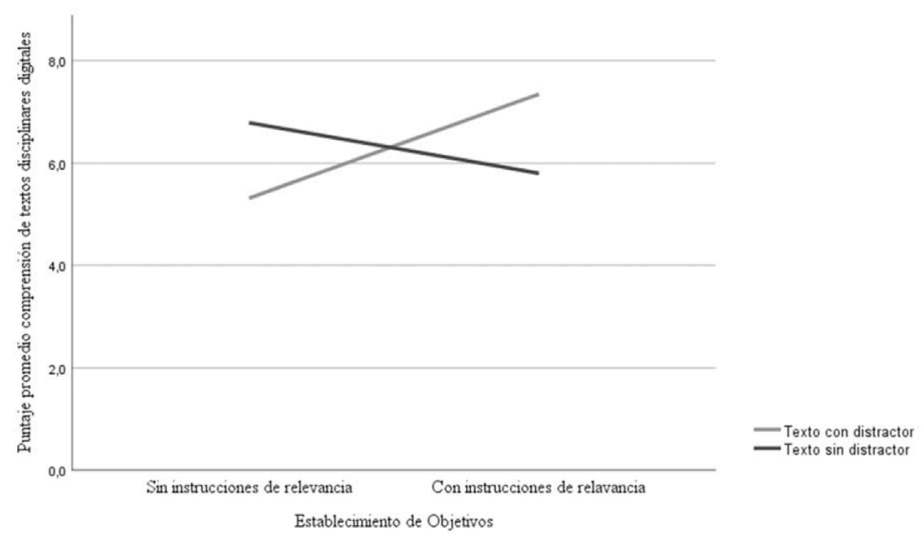


Figura 6 Efecto de las instrucciones sobre la comprensión de textos académicos digitales en condiciones de lectura con y sin distractores

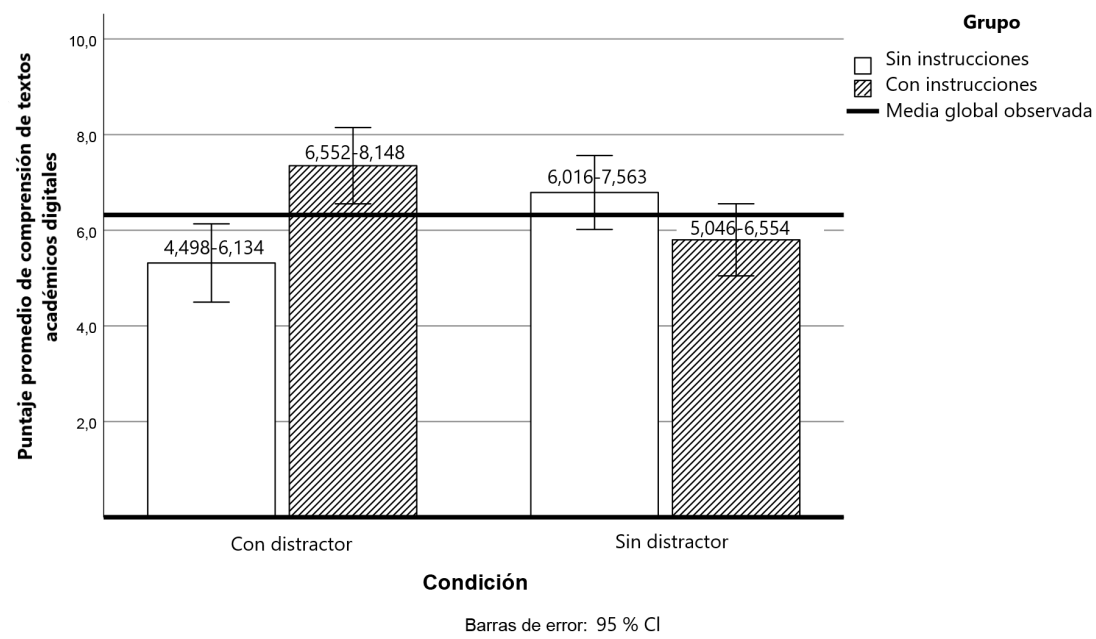

Es decir, al analizar los resultados obtenidos en las pruebas de lectura, en la condición de lectura con distractor, los estudiantes que son expuestos a instrucciones antes de leer obtienen puntajes promedio más altos en la prueba de lectura, que aquellos estudiantes que no son expuestos a instrucciones antes de leer. En cambio, en la condición sin distractor, disponer o no de instrucciones antes de leer un texto no origina diferencias estadísticamente significativas en la comprensión de textos disciplinares digitales (véase Figura 6).

\section{Discusión y conclusiones}

El objetivo de este estudio fue analizar el efecto de la inhibición y del establecimiento de objetivos en la comprensión de textos académicos digitales. Se esperaba encontrar diferencias estadísticamente significativas en el rendimiento en comprensión lectora en función de la capacidad de inhibición y de la capacidad de establecer objetivos de lectura a partir de instrucciones por los participantes. Se esperaba también hallar un efecto de interacción entre las variables, es decir, en la condición de lectura con distractores, los/as participantes que recibieron instrucciones de relevancia deberían obtener mejores resultados en la prueba de lectura que el grupo que no fue expuesto a ellas.
Los resultados muestran que existe un efecto de interacción entre la inhibición y el establecimiento de objetivos sobre la comprensión de textos académicos digitales, hallazgo que resulta coherente con la perspectiva de las FE como un constructo formado por procesos múltiples, que actúan de manera interrelacionada durante la ejecución de tareas novedosas o complejas (Anderson, 2002; Diamond, 2013; Friedman y Miyake, 2017).

Este resultado demuestra, de manera específica, que la ejecución de tareas novedosas o complejas, en este caso una condición de lectura de un texto académico digital que se ve interrumpida por distractores, propicia el despliegue de procesos múltiples, como el control de la interferencia y el despliegue de estrategias de organización o planificación de la lectura en función de un objetivo: para el control de la interferencia, mediante funciones de control inhibitorio como la inhibición de una respuesta impulsiva (mirar los distractores) o la resistencia a la interferencia distractora (suprimir la información que contenían los distractores); y en el caso del establecimiento de objetivos, por medio de la consideración de claves de relevancia (entregadas en las instrucciones) para el logro de un objetivo de lectura. De esta forma, obtener 
mejores resultados en la comprensión de un texto académico digital en un contexto de distracción cuando se presentan instrucciones antes de leer podría indicar que quien lee logra controlar y regular sus procesos cognitivos durante la lectura cuando la situación lo requiere.

A partir del resultado de este estudio es posible inferir que disponer de instrucciones antes de leer un texto académico digital en un contexto de distracción permite focalizar la actividad de lectura y evitar o disminuir el efecto que elementos distractores y ajenos a la tarea de lectura puede tener sobre el desempeño en comprensión. Este hallazgo es relevante, dado que la evidencia empírica ha demostrado que el rendimiento en comprensión de textos en formato digital es menor en comparación con el rendimiento lector a partir de textos en papel (Delgado et al., 2018; Piovano et al., 2018). En cambio, en ausencia de distractores, los lectores que probablemente sean más competentes para la lectura de un texto académico digital no se ven afectados ante la presencia o ausencia de instrucciones previas. Al respecto, una proyección del presente estudio sería considerar lectores en etapa escolar, con el fin de conocer si el efecto de las instrucciones en ausencia de distractores sería significativo, considerando que este tipo de lector(es) requiere de guías más directas para realizar sus tareas.

La presencia de instrucciones no produce diferencias estadísticamente significativas en el tiempo de mirada al distractor, lo que podría indicar que establecer objetivos de lectura a partir de instrucciones de relevancia no afecta la reacción ante un estímulo distractor. No obstante, el efecto de interacción detectado permite inferir que la influencia positiva de las instrucciones de relevancia en la comprensión de un texto académico podría explicarse principalmente por el efecto que estas tienen sobre el procesamiento del texto. Este hallazgo respalda la evidencia proveniente de investigaciones previas, que indican que ante la presencia de instrucciones, los lectores realizan un procesamiento focalizado y eficiente a partir de las claves de relevancia provistas por las instrucciones (Cerdán et al., 2013; Jian, 2018; León et al., 2019).

Un hallazgo que requiere mayor investigación es el que da cuenta del efecto del establecimiento de objetivos sobre la comprensión de un texto académico digital. Se esperaba encontrar un efecto principal de las instrucciones sobre la comprensión en las dos condiciones de lectura (con/sin distractor). No obstante, las instrucciones de relevancia parecen haber sido especialmente útiles solo cuando la tarea implicaba una carga cognitiva mayor, es decir, la situación de lectura que se ve interrumpida por distractores. Este resultado se alinea con investigaciones previas desarrolladas con estudiantes más jóvenes, donde la presencia de instrucciones, ayudas y guías específicas mejora los resultados de comprensión de lectura (Cerdán et al., 2019).

Dado que el tamaño muestral fue reducido por tratarse de un estudio piloto, experimentos con muestras más grandes podrían ayudar a definir si este efecto de interacción entre las instrucciones y los distractores se replica y consolida en las tareas de lectura a partir de textos académicos digitales. No obstante, pese a la limitación antes mencionada, la potencia observada para la interacción entre los factores permite realizar inferencias válidas en relación con las variables analizadas en este estudio. Por lo tanto, considerar un tamaño muestral mayor permitiría ampliar estos resultados y comprobar si existe un efecto principal de las variables inhibición y establecimiento de objetivos en la comprensión de textos académicos digitales.

En relación con el efecto de la variable inhibición sobre la comprensión, las diferencias detectadas no son significativas, lo que es coherente con investigaciones en las cuales se concluye que no en todos los estudios se comprueba la influencia de la inhibición en la comprensión lectora (Follmer, 2017; Roldán, 2016). No obstante, de acuerdo con la teoría de Friedman y Miyake (2004), se podría inferir que los(las) participantes de este 
estudio fueron capaces de resistir a la interferencia distractora, esto es, suprimir la información que contenían los distractores, dado que no lograron anular la respuesta automática (mirar al distractor). Esta inferencia se podría comprobar en estudios futuros, empleando una medida adicional de la inhibición.

Los resultados del presente estudio dan cuenta de la interrelación entre dos procesos que forman parte de la $\mathrm{FE}$ en función de su efecto en la comprensión de textos académicos. El estudio experimental reveló que leer instrucciones antes de leer un texto académico digital en un contexto en el cual la lectura se ve interrumpida por distractores tiene un efecto positivo significativo en la comprensión. No obstante, es necesario profundizar, en futuras investigaciones, en la explicación de estos hallazgos mediante el estudio del procesamiento del texto y las instrucciones, completando los datos reportados en este estudio con los patrones de secuencia de lectura del texto y las instrucciones, con el objetivo de contribuir a la necesidad de explicar cómo recibir distintos tipos de instrucciones de lectura afecta el procesamiento y la atención de los lectores a los distintos segmentos relevantes del texto.

\section{Agradecimientos}

Pamela Ramírez-Peña agradece a ANID-CONICYT por el financiamiento otorgado para cursar estudios de doctorado: CONICYT-PCHA/Doctorado nacional/2016-21161449

Claudia Pérez-Salas agradece a ANID-CONICYT, proyecto FONDECYT Regular 1181265, del que es investigadora responsable.

\section{Referencias}

Anderson, P. (2002). Assessment and development of executive function (EF) during childhood. Child Neuropsychology, 8(2), 71-82. https://doi.org/10.1076/ chin.8.2.71.8724

Annerer-Walcher, S., Korner, C., Beaty, R. E. y Benedek, M. (2020). Eye behavior predicts susceptibility to visual distraction during internally directed cognition.
Attention Perception \& Psychophysics, 82, 3432-3444. https://doi.org/10.3758/s13414-020-02068-1

Arrington, C. N., Kulesz, P. A., Francis, D. J., Fletcher, J. M. y Barnes, M. A. (2014). The contribution of attentional control and working memory to reading comprehension and decoding. Scientific Studies of Reading, 18(5), 325-346. http://dx.doi.org/10.108 $0 / 10888438.2014 .902461$

Balluerka, N. y Vergara, A. I. (2002). Diseños de investigación experimental en psicología. Pearson.

Borella, E., Carreti, B. y Pelegrina, S. (2010). The specific role of inhibition in reading comprehension in good and poor comprehenders. Journal of Learning Disabilities, 43(6), 541-552. https://doi. org/10.1177/0022219410371676

Borella, E. y de Ribaupierre, A. (2014). The role of working memory, inhibition, and processing speed in text comprehension in children. Learning and Individual Differences, 34, 86-92. https://doi.org/10.1016/j. lindif.2014.05.001

Canet-Juric, L., Burin, D., Andrés, M. L. y Urquijo, S. (2013). Perfil cognitivo de niños con bajos rendimientos en comprensión lectora. Anales de Psicologia, 29(3), 996-1005. https://doi.org/10.6018/ analesps.29.3.138221

Canet-Juric, L., Urquijo, S., Richard's, M. y Burin, D. (2009). Predictores cognitivos de niveles de comprensión lectora mediante análisis discriminante. International Journal of Psychological Research, 2(2), 99-111. https://doi.org/10.21500/20112084.865

Cerdán, R., Marín, M. y Candel, C. (2013). The role of perspective on students' use of multiple documents to solve an open-ended task. Psicología Educativa, 19(2), 89-94. https://doi.org/10.1016/ S1135-755X(13)70015-0

Cerdán, R., Pérez, A., Vidal-Abarca, E. y Rouet, J. F. (2019). To answer questions from text, one has to understand what the question is asking: Differential effects of question aids as a function of comprehension skill. Reading and Writing, 32, 2111-2124. https://doi. org/10.1007/s11145-019-09943-w

De Beni, R., Palladino, P., Pazzaglia, F. y Cornoldi, C. (1998). Increases in intrusion errors and working memory deficit of poor comprehenders. The Quarterly Journal of Experimental Psychology A: Human Experimental Psychology, 51(2), 305-320. https:// doi.org/10.1080/713755761

Delgado, P., Vargas, C., Ackerman, R. y Salmeron, L. (2018). Don't throw away your printed books: 
A meta-analysis on the effects of reading media on reading comprehension. Educational Research Review, 25, 23-38. https//doi.org/10.1016/j. edurev.2018.09.003

Demagistri, M. S., Richards, M. M. y Canet Juric, L. (2014). Incidence of executive functions on reading comprehension performance in adolescents. Electronic Journal of Research in Educational Psychology, 12(2), 343-370. https:// doi.org/10.25115/ejrep.33.13146

Diamond, A. (2013). Executive functions. Annual Review of Psychology, 64, 135-168. https://doi.org/10.1146/ annurev-psych-113011-143750

Follmer, D. J. (2017). Executive function and reading comprehension: A meta-analytic review. Educational Psychologist, 53(1), 42-60. https://doi.org/10.1080 /00461520.2017.1309295

Friedman, N. P. y Miyake, A. (2004). The relations among inhibition and interference control functions: A latent-variable analysis. Journal of Experimental Psychology: General, 133(1), 101-135. https://doi. org/10.1037/0096-3445.133.1.101

Friedman, N. P. y Miyake, A. (2017). Unity and diversity of executive functions: Individual differences as a window on cognitive structure. Cortex, 86, 186-204. https://doi.org/10.1016/j.cortex.2016.04.023

Georgiou, G. y Das, J .P. (2016). What component of executive functions contributes to normal and impaired reading comprehension in young adults? Research in Developmental Disabilities, 49(50), 118-128. https://doi.org/10.1016/j.ridd.2015.12.001

Gernsbacher, M. A. (1993). Less skilled readers have less efficient suppression mechanisms. Psychological Science, 4(5), 294-298. https://doi. org/10.1111/j.1467-9280.1993.tb00567.x

Gaspelin, N. y Luck, S. J. (2018). The role of inhibition in avoiding distraction by salient stimuli. Trends in Cognitive Sciences, 22(1), 79-92. https://doi. org/10.1016/j.tics.2017.11.001

Jian, Y.-C. (2018). Reading instructions facilitate signaling effect on science text for young readers: An eyemovement study. International Journal of Science and Mathematics Education, 17, 503-522. https:// doi.org/10.1007/s10763-018-9878-y

Kintsch, W. (1998). Comprehension. A paradigm for cognition. Cambridge University Press.

León, J. A., Moreno, J. D., Escudero, I., Olmos, R., Ruiz, M. y Lorch, R. (2019). Specific relevance instructions promote selective reading strategies: Evidences from eye tracking and oral summaries. Journal of Research in Reading, 42(2), 432-453. https://doi. org/10.1111/1467-9817.12276

McCrudden, M. (2011). Do specific relevance instructions promote transfer appropriate processing? Instructional Science, 39(6), 865-879. https://doi. org/10.1007/s11251-010-9158-x

McCrudden, M. y Schraw, G. (2007). Relevance and goalfocusing in text processing. Educational Psychology Review, 19(2), 113-139. https://doi.org/10.1007/ s10648-006-9010-7

Parodi, G., Moreno-de-León, T. y Julio, C. (2020). Comprensión de textos escritos: reconceptualizaciones en torno a las demandas del siglo XxI. Íkala, Revista de Lenguaje y Cultura, 25(3), 775-795. https://doi. org/10.17533/udea.ikala.v25n03a10

Piovano, S., Irrazabal, N. y Burin, D. I. (2018). Comprensión de textos expositivos académicos en e-book Reader y en papel: influencia del conocimiento previo de dominio y la aptitud verbal. Ciencias Psicológicas, 12(2), 177-185. https://doi.org/10.22235/cp.v12i2.1680

Potocki, A., Sanchez, M., Ecalle, J. y Magnan, A. (2017). Linguistic and cognitive profiles of 8- to 15-yearold children with specific reading comprehension difficulties: The role of executive functions. Journal of Learning Disabilities, 50(2), 128-42. https://doi. org/10.1177/0022219415613080

Ramírez-Peña, P. (2020). Funciones ejecutivas y comprensión de textos académicos en estudiantes de primer año [Tesis de doctorado, Universidad de Concepción]. http://repositorio.udec.cl/xmlui/ handle/11594/4667

Rapp, D. N. y McCrudden, M. T. (2018). Relevance before, during, and after discourse experiences. In A. Schüller-Zwierlein (Ed.), Relevance and irrelevance: Theories, factors and challenges (pp. 141-160). De Gruyter Saur. https://doi. org/10.1515/9783110472509-007

Riffo, B., Véliz, M., Reyes, F., Castro, G., Reyes, A. y Campos, D. (2013). Evaluación de la comprensión lectora. Desarrollo de un instrumento basado en un modelo psicolingüistico. Universidad de Concepción.

Roldán, L. A. (2016). Inhibición y actualización en comprensión de textos: una revisión. Universitas Psychologica, 15(2), 87-96. https://doi. org/10.11144/Javeriana.upsy15-2.iact

Rozek, E., Kemper, S. y McDowd, J. (2012). Learning to ignore distracters. Psychology and Aging, 27(1), 61-66. https://doi.org/10.1037/a0025578 
Salmerón, L. y Delgado, P. (2019). Critical analysis of the effects of the digital technologies on reading and learning / Análisis crítico sobre los efectos de las tecnologías digitales en la lectura y el aprendizaje. Culture and Education, 31(3), 465-480. https:// doi.org/10.1080/11356405.2019.1630958

Snow, C. (2002). Reading for understanding. Toward an R\&D program in reading comprehension. RAND Corporation.

Véliz, M., Riffo, B. y Salazar, O. (2013a). Lectum 7. Prueba de comprensión lectora, forma $A$. Universidad de Concepción.

Véliz, M., Riffo, B. y Salazar, O. (2013b). Lectum 7. Prueba de comprensión lectora, forma $B$. Universidad de Concepción.
Vellutino, F. R., Tunmer, W. E., Jaccard, J.J.y Chen, R. (2007). Components of reading ability: Multivariate evidence for a Convergent Skills Model of Reading Development. Scientific Studies of Reading, 11(1), 3-32. https://doi.org/10.1080/10888430709336632

Vidal-Abarca, E., Salmeron, L. y Mañá, A. (2011). Individual differences in task-oriented reading. In M. T. McCrudden, J. P. Magliano y G. Schraw (Eds.), Text relevance and learning from text (pp. 267-293). Information Age Publishing, Inc.

Wilcockson, T. D. W., Mardanbegi, D., Sawyer, P., Gellerser, H., Xia, B. Q. y Crawford, T. J. (2019). Oculomotor and inhibitory control in dyslexia. Frontiers in Systems Neuroscience, 12. https://doi.org/10.3389/ fnsys.2018.00066

Cómo citar este artículo: Ramírez-Peña, P., Pérez-Salas, C., Riffo, B., y Cerdán, R. (2022). Leer en contextos de distracción: el rol de la inhibición y el establecimiento de objetivos en la comprensión de textos académicos digitales. Íkala, Revista de Lenguaje y Cultura, 27(1), 66-83. https://doi.org/10.17533/ udea.ikala.v27n1a04 